\title{
TELEMEDICINA COMO FERRAMENTA DE ENSINO NO CUIDADO AO PACIENTE QUEIMADO
}

\author{
Telemedicine as a Teaching Tool in the Care of Burned Patients
}

\begin{abstract}
Alcir Escocia Dorigatti'; Flavio Nadruz Novaes²; Bruno Monteiro Tavares Pereira³; Mateus de Paula Solino4; Antonio Carlos da Silva II5; Armando Carlos Franco de Godoy ${ }^{6}$; Gustavo Pereira Fraga ${ }^{7}$
\end{abstract}

Resumo No contexto da saúde e educação médica, o cuidado ao paciente queimado ocupa uma pequena carga horária na formação. Objetivos: Avaliar os conhecimentos no cuidado do paciente queimado e validar o uso da telemedicina como instrumento de propagação destes conhecimentos. Materiais e Métodos: Aplicou-se dois questionários iguais com 10 questões, online, via SurveyMonkey, antes e após curso com 8 aulas de uma hora de duração cada, presencial ou via videoconferência. Ao final do curso uma prova online com 36 questões foi realizada. Resultados: Participaram do curso gratuito 215 pessoas, sendo $53 \%$ estudantes, $23 \%$ enfermeiros, $9 \%$ técnicos e $6 \%$ residentes. Desses, 39\% já haviam participado do atendimento ao paciente queimado e $22 \%$ haviam realizado curso de formação. Dos 69 que concluíram o curso, $68,1 \%$ acompanharam presencialmente, enquanto $31,9 \%$ via telemedicina. No teste aplicado antes do curso a média foi de $56,6 \%$ de acertos e após o curso $84,8 \%$. A nota final atribuída ao curso para aqueles que o concluíram foi de 8,6 (máxima de 10). Conclusão: A realização de palestras via telemedicina é ferramenta útil na propagação dos conceitos no cuidado a saúde.

Palavras-chave: Queimaduras; Educação continuada; Telemedicina.

\begin{abstract}
In the context of health care and medical education, burned patient's care takes a small workload in training. Aims: To assess the knowledge in burn care and to validate the use of telemedicine as a tool to spread this knowledge. Materials and Methods: Two identical questionnaires were provided with 10 questions online via SurveyMonkey before and after an 8 lessons course with one hour each, live or through videoconference. At the end of the course an online test with 36 questions was conducted. Results: A total of 215 people attended the free-of-charge course. 53\% students, $23 \%$ nurses, $9 \%$ technicians and $6 \%$ residents. Of these, 39\% participated in some extent to the care of burned patients and $22 \%$ have attended training courses in the past. Of the 69 who completed the course, $68.1 \%$ attended in person, while $31.9 \%$ through telemedicine. In the test performed before the course, the average rate was $56.6 \%$ correct and after the course 84.8 $\%$. The final score of the course for those who have completed was 8.6 (out of 10). Conclusion: Lectures through telemedicine are a useful tool in spreading the concepts in health care.
\end{abstract}

Keywords: Burns; Continuing Education; Telemedicine.

\footnotetext{
1.Acadêmico do curso de Medicina, Faculdade de Ciências Médicas (FCM), Universidade Estadual de Campinas (Unicamp), Campinas, SP, Brasil; 2. Membro Titular da Sociedade Brasileira de Queimaduras e da Sociedade Brasileira de Cirurgia Plástica; Coordenador da Unidade de Tratamento de Queimaduras da Santa Casa de Limeira, Limeira, SP, Brasil; 3. Médico Assistente da Disciplina de Cirurgia do Trauma do Departamento de Cirurgia, Faculdade de Ciências Médicas, Universidade Estadual de Campinas (Unicamp), Campinas, SP, Brasil; 4. Acadêmico do curso de Medicina, Faculdade de Ciências Médicas (FCM), Universidade Estadual de Campinas (Unicamp), Campinas, SP, Brasil. 5. Analista de Suporte e Redes da Divisão de Informática do Hospital de Clínicas da Unicamp, Campinas, SP, Brasil; 6. Professor, Fisioterapeuta da Unidade de Terapia Intensiva Adulto do Hospital de Clínicas da Unicamp, Campinas, SP, Brasil; 7. Professor Coordenador da Disciplina de Cirurgia do Trauma do Departamento de Cirurgia, Faculdade de Ciências Médicas, Universidade Estadual de Campinas (Unicamp), Campinas, SP, Brasil.
} 


\section{Introdução}

O Advanced Burn Life Support (ABLS) é um curso realizado em um dia pela Associação Americana de Queimaduras (American Burn Association - ABA) para médicos, enfermeiros e demais profissionais da saúde. $O$ curso fornece subsídios para tratar pacientes durante as primeiras 24 horas após a lesão. A ABA desenvolveu um curso baseado na web, no qual busca fazer o mesmo, o ABLS-Now'.

Nesta mesma ideologia, a Sociedade Brasileira de Queimaduras possui o Curso Nacional de Normatização de Atendimento ao Queimado (CNNAQ), realizado em oito módulos em um único dia, com foco para profissionais de saúde, visando a atualizar os conhecimentos em atendimento ao paciente queimado. Entretanto, ainda não há um programa fixo de ensino a distância para este curso ${ }^{2}$.

Com o objetivo de ampliar a divulgação do atendimento inicial ao paciente queimado e promover educação continuada, a Telemedicina representa um potencial método que utiliza a tecnologia de telecomunicação para assegurar serviços e educação em saúde à distância. Tais avanços tecnológicos são realidade e já permitem que educação e cuidado ao paciente em seu maior estado-da-arte ou excelência sejam alcançados por especialistas em diferentes localizações do Globo $^{3,4}$. A videoconferência é um dos instrumentos da Telemedicina e tem se apresentado de forma muito útil quando empregada para a educação a distância, sendo possível realizar colaborações com tomada de decisão em tempo real, assim como levar conhecimento a profissionais que não possam estar em um mesmo espaço físico ${ }^{5}$. No Brasil, o Governo Federal, através do Ministério de Ciência e Tecnologia, investiu na criação de uma rede de Telemedicina chamada RUTE (Rede Universitária de Telemedicina) ${ }^{6}$. Esta rede vem conectando diversos hospitais universitários no país, incluindo todas suas especialidades, em uma única rede de informação e comunicação.
Desta forma, a Telemedicina não só facilita o acesso à informação, mas é também um meio de comunicação em grupo de custo relativamente baixo que possibilita sessões científicas e interdisciplinares dentro dos serviços de saúde, isentando assim a necessidade de deslocamento de professores e alunos e permitindo maior flexibilidade quanto ao tempo. A Telemedicina permite incorporar atividades de educação e de administração em saúde, além de apresentar características de versatilidade podendo ser utilizada em diferentes cenários, seja numa realidade civil ou militar, seja em cenários austeros e catastróficos ${ }^{3,47-14}$.

A necessidade de expandir o conhecimento no atendimento inicial de queimados na região metropolitana de Campinas, somada à escassez de centros especialistas em tratamento de queimados nesta geografia, serviram como racional para realização do CNNAQ em um hospital-escola capacitado para transmitir aulas por telemedicina, unindo assim a versatilidade e o alcance da Telemedicina com o conteúdo distinto do curso CNNAQ. Por fim, os autores elaboraram a hipótese de que seria possível a execução de um curso a distância para profissionais da área de saúde, avaliando posteriormente os conhecimentos adquiridos no cuidado do paciente queimado nas primeiras 24 horas do atendimento, validando assim o uso da telemedicina e web como instrumentos úteis na propagação destes conhecimentos.

\section{Métodos}

O Hospital de Clínicas (HC) da Universidade Estadual de Campinas (Unicamp) é um hospital universitário localizado em Campinas, São Paulo, com 420 leitos hospitalares e atendimento em todas as especialidades clínicas e cirúrgicas. A área de cobertura do serviço inclui toda a Região Metropolitana de Campinas, com aproximadamente três milhões de habitantes.

Através do projeto "Professor Especialista Visitante da Pró-Reitoria de Graduação da Unicamp", surgiu a pos- 
sibilidade de receber no HC-Unicamp, um profissional (o autor Flavio Nadruz Novaes) com grande experiência em queimaduras, atuando com carga horária de 8 horas semanais, durante cinco meses, no segundo semestre de 2013. O curso, criado pela Sociedade Brasileira de Queimaduras (SBQ) e apoiado pela Sociedade Brasileira de Atendimento Integrado ao Traumatizado (SBAIT), tem a finalidade de promover o conhecimento e as normas aos profissionais de saúde que prestam o primeiro atendimento às vítimas de queimadura de qualquer etiologia, a fim de uniformizar as condutas de atendimento emergencial ao queimado durante as primeiras 24 horas do atendimento ${ }^{2}$.

O curso, livre de qualquer tarifa, teve uma rápida divulgação (Figura 1) na instituição sede, pela SBQ, SBAIT e pela rede RUTE. A inscrição no curso estava vinculada à realização de um questionário online via SurveyMonkey. Ao realizar a inscrição os alunos obrigatoriamente respondiam um pré-teste com 10 questões. Ao final do curso as mesmas 10 questões eram novamente aplicadas em conjunto com outras 26 questões, totalizando

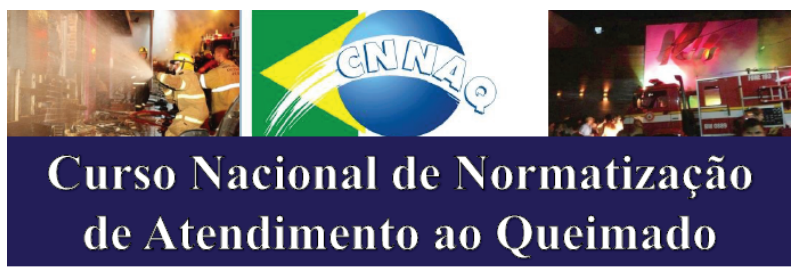

Data: Terças feiras (agosto a outubro de 2013) - 12 às $13 \mathrm{~h}$. Local: Sala de Telemedicina $-3^{\circ}$ andar HC Unicamp

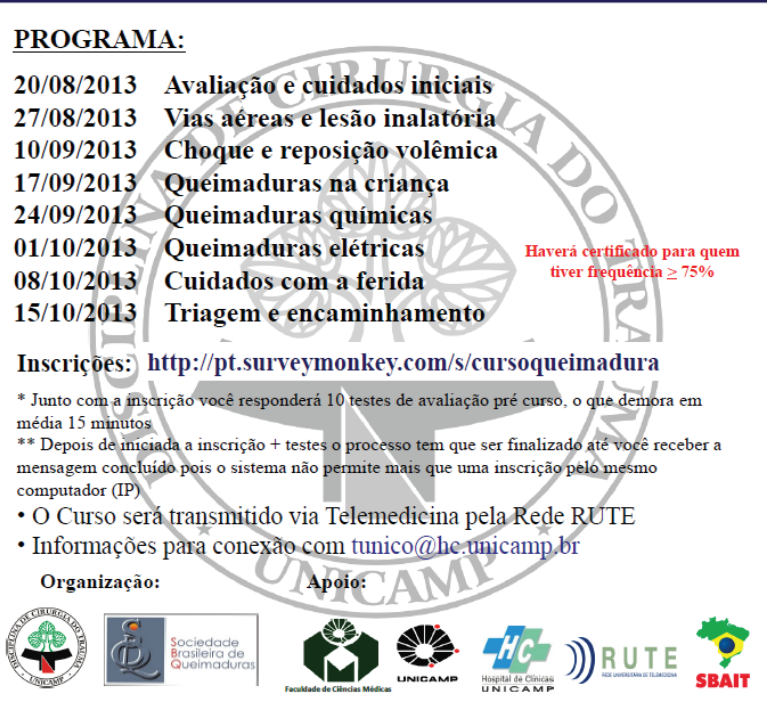

Figura 1. Cartaz de divulgação do curso

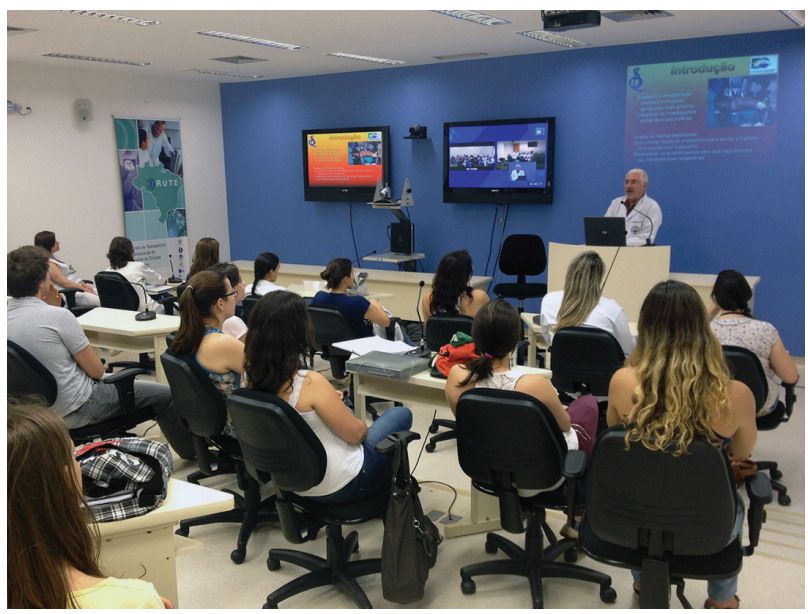

Figura 2. Aula do curso na sala de telemedicina do HC-Unicamp.

uma prova final com 36 questões. Nos resultados descritos abaixo, "pré-teste" se refere às 10 questões aplicadas no momento da inscrição; "pós-teste" às respostas dos mesmos 10 testes na prova final e "nota final/média final" como a nota na prova com 36 questões.

No total foram realizadas e transmitidas 8 aulas com uma hora de duração cada uma, de agosto a outubro de 2013, com os seguintes temas: Avaliação e cuidados iniciais; vias aéreas e lesão inalatória; choque e reposição volêmica; queimaduras na criança; queimaduras químicas; queimaduras elétricas; cuidados com a ferida; triagem e encaminhamento. Nas duas semanas finais do curso os alunos receberam uma apostila do CNNAQ, em PDF, com o conteúdo ensinado nas aulas para que estudassem mais sobre os temas. A avaliação final só foi realizada por alunos que tiveram a frequência mínima de $75 \%$.

Os dados gerados foram tabulados, utilizando o software "Microsoft Excel ${ }^{\circledR}$, e posteriormente analisados. Foram excluídas das análises estatísticas as respostas deixadas em branco.

Foram avaliadas as características demográficas dos participantes, assim como a evolução de notas baseadas nas 10 questões respondidas duas vezes e em sua nota final na prova com 36 questões.

O curso pôde ser acompanhado em diversos centros no país, através da Telemedicina, pelo sistema RUTE e via web (Figura 2$)^{6}$. 


\section{Resultados}

Realizaram a inscrição gratuita para o curso 205 pessoas, mas apenas 69 pessoas o concluíram e fizeram a prova final. Já haviam participado do atendimento ao paciente queimado $39 \%$ dos inscritos e $22 \%$ já haviam realizado algum curso de formação.

Os participantes a distância que se conectaram através da telemedicina pertenciam às seguintes localidades: FURGS (Rio Grande - RS), Universidade Estadual de Londrina - UEL (Londrina - PR), Hospital Municipal Dr. Mario Gatti (Campinas - SP) e Instituto Nacional de Traumatologia e Ortopedia (Rio de Janeiro - RJ), além de outros alunos que acompanharam o curso através da web. Dessa forma, o curso conseguiu atingir quatro estados diferentes ao mesmo tempo.

Daqueles que se matricularam, 53\% eram acadêmi$\cos , 23 \%$ enfermeiros, $9 \%$ técnicos e $6 \%$ médicos residentes. O perfil daqueles que concluíram o curso foi $72 \%$ de acadêmicos, $10 \%$ de enfermeiros, $4 \%$ de técnicos, $1,5 \%$ de médicos residentes e $1,5 \%$ de técnicos.

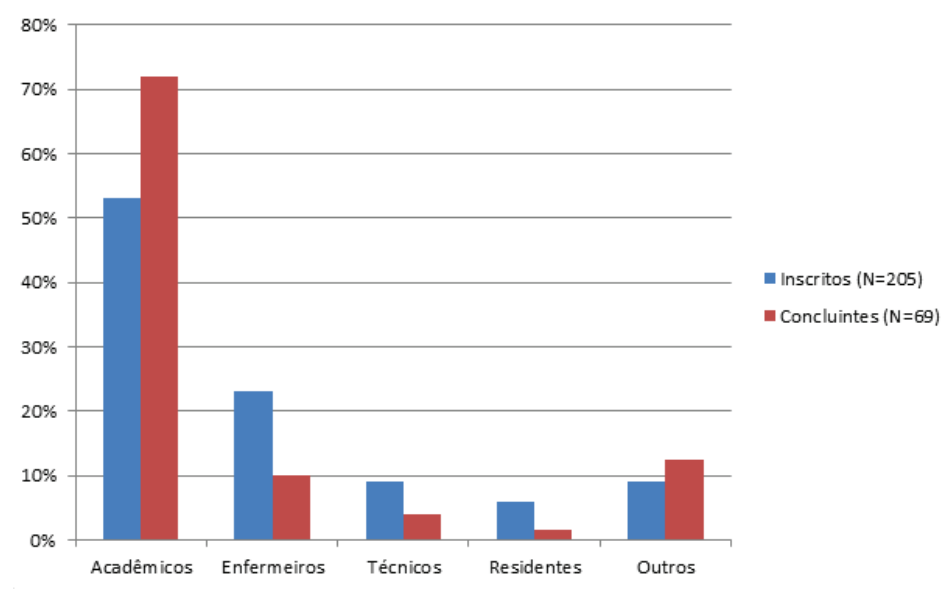

Gráfico 1. Perfil profissional dos inscritos e concluintes do curso.

Daqueles que concluíram o curso, 69,1\% acompanharam presencialmente, enquanto $30,9 \%$ o fizeram via telemedicina ou web. Entre os que assistiram presencialmente, $71,7 \%$ tiveram apenas uma ou nenhuma falta, contra $68,4 \%$ entre os que acompanharam via telemedicina.
No pré-teste aplicado antes da realização do curso a média para aqueles que terminaram o curso foi de $59,2 \%$ de acertos e após o curso passou para 83,5\%. Já a média final entre os que participaram presencialmente foi de $86,7 \%$, contra $58,3 \%$ dos que participaram via telemedicina.

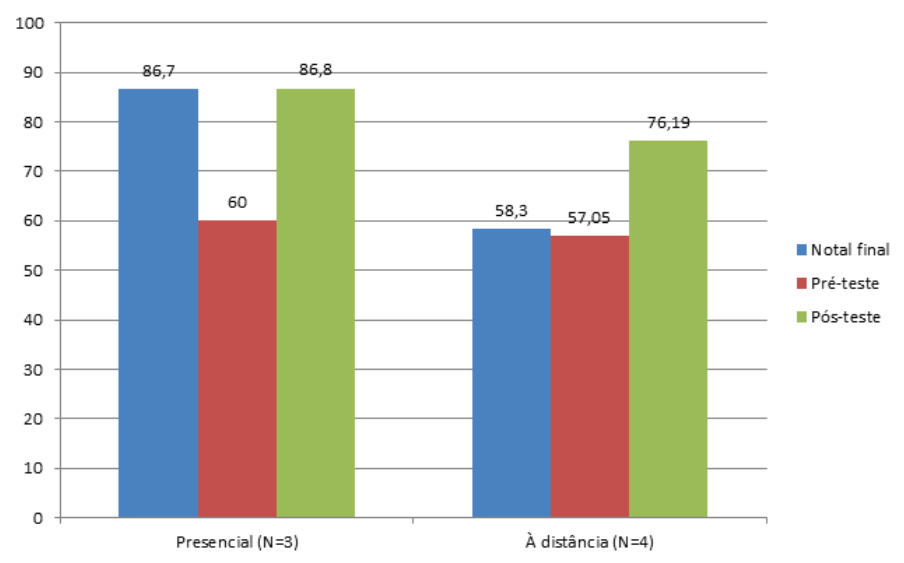

Gráfico 2. Comparação de desempenho entre os que fizeram o curso fisicamente e remotamente.

A nota final atribuída ao curso por aqueles que o concluíram foi de 8,6 de 10 .

\section{Discussão}

A Telemedicina já é considerada uma boa ferramenta quando utilizada para o ensino ${ }^{3-14}$. Ao permitir maior acessibilidade e flexibilidade, ela torna-se meio de divulgação sem fronteiras e independente de fatores geográficos ${ }^{15,16}$. Isto foi observado na participação de profissionais de diferentes cidades que acompanharam o curso através de videoconferência. Constatou-se que no momento da inscrição, diversos profissionais manifestaram interesse em realizar o curso, embora ocorresse a predominância de acadêmicos. Tal fato pode ser justificado pelo curso ter ocorrido em um ambiente universitário o que facilita e favorece o acesso de tal grupo. Entretanto, houve uma grande queda no número de concluintes em relação ao número de inscritos no início e nas primeiras aulas do curso. Tal queda foi mais significante em todas as categorias profissionais, exceto para 
os acadêmicos que apresentaram um aumento relativo em sua participação, pois outras categorias tiveram mais desistências em realizar o curso que os acadêmicos. Este fato pode ser explicado pela longa duração do curso (8 semanas). O CNNAQ normalmente ocorre em um dia, todavia os autores optaram por diluir o mesmo conteúdo em 8 aulas de uma hora, de forma a aumentar a participação no curso. É possível que devido às atividades de trabalho, tal organização tenha impedido que muitos profissionais de fato realizassem o programa do curso, dado o número extenso e repartido de aulas. A realização do CNNAQ através da Telemedicina foi uma iniciativa pioneira até então não descrita e outros formatos de como ofertá-lo podem ser desenvolvidos de maneira a aprimorar o alcance, qualidade e frequência do mesmo.

Observou-se neste estudo, diferença significativa entre o desempenho final quando comparamos os alunos que realizaram o curso presencialmente ou remotamente. Tal achado não pode ser justificado exclusivamente pela frequência em aulas, pois embora exista relação entre a frequência nas aulas e o desempenho, a presença foi semelhante para ambos os grupos. Talvez os participantes do curso presencial tenham sido mais estimulados para realizarem estudo complementar com a apostila enviada aos participantes.

Entretanto, quando comparada a evolução entre os pré-testes e pós-testes, todas as categorias apresentaram melhora no desempenho. Houve um aumento de $24,3 \%$ no desempenho médio de todos os alunos que fizeram o curso até o fim. Este achado confirma o uso da telemedicina como instrumento eficaz para o ensino e abre novas possibilidades para cursos nacionais de normatização, como o CNNAQ, ao permitir o fácil acesso a cursos de atualização em qualquer lugar do país que possua o sistema de telemedicina ou acesso a web.

Outro ponto importante ao se realizar um curso sob uma nova metodologia de ensino é o quanto os alunos consideraram o curso eficaz. O presente curso obteve boa aprovação dos alunos que o fizeram, mesmo sendo o primeiro realizado no formato apresentado. Este resultado incentiva o desenvolvimento de novas iniciativas com a utilização deste método e a reflexão e aprimoramento do que foi realizado de forma a instituir este novo formato de maneira definitiva aos quadros do ensino de atendimento ao paciente queimado. Outros estudos de cunho internacional já demonstraram a aprovação e resultados de iniciativas semelhantes, de forma a complementar a educação em queimaduras e outras especialidades através de sistemas baseados na web, o que configura outra possibilidade de modelo para o curso ${ }^{17-19}$.

Este foi o primeiro estudo envolvendo os conceitos do atendimento inicial ao queimado (CNNAQ) e educação a distância via telemedicina em âmbito nacional. Os bons resultados apresentados nesta série demonstram que a educação médica no Brasil pode ser contínua, de baixo custo e com bom aproveitamento por parte do aluno, especificamente em se tratando do atendimento ao paciente queimado, assunto ainda pouco ensinado nos currículos dos cursos de graduação nas áreas de saúde.

\section{Conclusão}

A realização de curso sobre queimaduras presencial e a distância via telemedicina mostrou ser útil na formação de alunos e profissionais, que tiveram melhora do desempenho em testes realizados antes e após o curso.

\section{Referências}

1. A Self-Directed, Web-Based Learning Program produced by the American Burn Association. Acessado em 5 de Dezembro de 2013 em: http://www.ameriburn.org/ ABLS/ABLSNow.htm

2. CNNAQ - Curso Nacional de Normatização de Atendimento ao Queimado. Acessado em 5 de Dezembro de 2013 em: http://sbqueimaduras.org.br/cnnaq-curso-nacionalde-normatizacao-de-atendimento-ao-queimado

3. Marttos AC, Kuchkarian FM, Abreu-Reis P, Pereira BMT, Collet-Silva FS, Fraga GP. World J Emerg Surgy. 2012; 7(Suppl 1):S4 
4. Pereira BMT, Calderan TRA, Nolasco da Silva MT, Carlos da Silva A, Marttos Jr AC, Fraga GP. Initial experience at a university teaching hospital from using telemedicine to promote education through video conferencing. Sao Paulo Med J. 2012; 130(1):32-6.

5. Hays RB, Peterson L. Options in education for advanced trainees in isolated general practice. Aust Fam Physician. 1996;25(3):362-366.

6. Rede Universitária de Telemedicina. Acessado em 5 de Dezembro de 2013 em: http://rute.rnp.br

7. American Telemedicine Association: Telemedicine Defined. Accessed on December 5th, 2013, from http://www.americantelemed.org/i4a/pages/index.cfm?pageid=3333

8. Ereso $A Q$, Garcia P, Tseng E, Gauger G, Kim H, Dua MM, et al. Live transference of surgical subspecialty skills using telerobotic proctoring to remote general surgeons. J Am Coll Surg. 2010; 211(3):400-11.

9. Mauer UM, Kunz U. Management of neurotrauma by surgeons and orthopedists in a military operational setting. Neurosurg Focus. 2010;28(5):E10.

10. Huang CM, Chan E, Hyder AA. Web 2.0 and internet social networking: a new tool for disaster management? - lessons from Taiwan. BMC Med Inform Decis Mak. 2010; 6:10:57.

11. Blanche PA, Bablumian A, Voorakaranam R, Christenson C, Lin W, Gu T, et al. Holographic three-dimensional telepresence using large-area photorefractive polymer. Nature. 2010; 468(7320):80-3.
12. Jabbour P, Gonzalez LF, Tjoumakaris S, Randazzo C, Rosenwasser R. Stroke in the robotic era. World Neurosurg. 2010; 73(6):603-4.

13. Latifi R, StanonikMde L, Merrell RC, Weinstein RS. Telemedicine in extreme conditions: supporting the Martin Strel Amazon Swim Expedition. Telemed J E Health. 2009; 15(1):93-100.

14. Landers SH. Why health care is going home. N Engl J Med. 2010; 363(18):1690-1.

15. Hede K. Teleoncology gaining acceptance with physicians, patients. J Natl Cance Inst. 2010; 102(20):1531-3.

16. García Jordá E. Telemedicine: shortening distances. Clin Transl Oncol. 2010 12(10):650-1.

17. Cochran A, Edelman LS, Morris SE, Saffle JR. Learner Satisfaction With Web-Based Learning as an Adjunct to Clinical Experience in Burn Surgery. J Burn Care Res 2008; $29 \cdot 222-6$.

18. Chumley-Jones HS, Dobbie A, Alford CL. Web-based learning: sound educational method or hype? A review of the evaluation literature. Acad Med 2002; (10, Suppl):S86-S93.

19. Curran V, Hoekman T, Gulliver W, Landells I, Hatcher L. Web-based continuing medical education. II. Evaluation study of computer-mediated continuing medical education. J Contin Educ Health Prof. 2000; 20:106-19. 